\title{
Spectral characteristics of Mrk 501 during the 2012 and 2014 flaring states
}

\author{
Gabriele Cologna* ${ }^{*} \dagger$, Nachiketa Chakraborty ${ }^{b}$, Mahmoud Mohamed ${ }^{a \dagger}$, Frank Rieger ${ }^{b}$, \\ Carlo Romoli ${ }^{c}$, Andrew Taylor ${ }^{c}$, Stefan J. Wagner ${ }^{a}$, Alicja Wierzcholska ${ }^{a, d}{ }^{\ddagger}$, Agnieszka \\ Jacholkowska $^{e}$ for the H.E.S.S. Collaboration and Omar Kurtanidze ${ }^{f, a}$ \\ ${ }^{a}$ Landessternwarte, Universität Heidelberg, Königstuhl, D 69117 Heidelberg, Germany \\ ${ }^{b}$ Max-Planck-Institut für Kernphysik, P.O. Box 103980, D 69029 Heidelberg, Germany \\ ${ }^{c}$ Dublin Institute for Advanced Studies, 31 Fitzwilliam Place, Dublin 2, Ireland \\ ${ }^{d}$ Insitute of Nuclear Physics, Polish Academy of Science, ul. Radzikowskiego 152, 31-342 \\ Kraków, Poland \\ e LPNHE, Université Pierre et Marie Curie Paris 6, Université Denis Diderot Paris 7. \\ CNRS/IN2P3, 4 Place Jussieu, F-75252, Paris Cedex 5, France \\ ${ }^{f}$ Abastumani Observatory, Mt. Kanobili, 0301 Abastumani, Georgia \\ E-mail: gcologna@lsw.uni-heidelberg.de
}

\begin{abstract}
Observations at Very High Energies (VHE, E > $100 \mathrm{GeV}$ ) of the BL Lac object Mrk 501 taken with the High Energy Stereoscopic System (H.E.S.S.) in four distinct periods between 2004 and 2014 are presented, with focus on the 2012 and 2014 flaring states. The source is detected with high significance above $\sim 2 \mathrm{TeV}$ in $\sim 13.1 \mathrm{~h}$ livetime. The observations comprise low flux states and strong flaring events, which in 2014 show a flux level comparable to the 1997 historical maximum. Such high flux states enable spectral variability and flux variability studies down to a timescale of four minutes in the $2-20 \mathrm{TeV}$ energy range. During the 2014 flare, the source is clearly detected in each of these bins. The intrinsic spectrum is well described by a power law of index $\Gamma=2.15 \pm 0.06$ and does not show curvature in this energy range. Flux dependent spectral analyses show a clear harder-when-brighter behaviour. The high flux levels and the high sensitivity of H.E.S.S. allow studies in the unprecedented combination of short timescales and an energy coverage that extends significantly above $10 \mathrm{TeV}$. The high energies allow us to probe the effect of EBL absorption at low redshifts, jet physics and LIV. The multiwavelength context of these VHE observations is presented as well.
\end{abstract}

The 34th International Cosmic Ray Conference,

30 July- 6 August, 2015

The Hague, The Netherlands

\footnotetext{
*Speaker.

${ }^{\dagger}$ Member of the International Max Planck Research School for Astronomy and Cosmic Physics at the University of Heidelberg (IMPRS-HD) and the Heidelberg Graduate School of Fundamental Physics (HGSFP).

${ }^{\ddagger}$ Mobility Plus Fellow
} 


\section{Introduction}

With the exception of two starburst galaxies and a few sources in the Large Magellanic Cloud, active galactic nuclei (AGN) are up to now the sole detected extragalactic TeV emitters. Despite several classes of AGN existing, the majority of the detections in this energy range consists of blazars, in particular the subclass of high frequency peaked BL Lac objects (HBL). Mrk 501 is one of the nearest HBL, with a redshift $z \sim 0.034$, and was the second extragalactic object to be detected as Very High Energy (VHE, E $>100 \mathrm{GeV}$ ) emitter in 1995 [1]. It is known for its strong variability at all energies and is referred to as an extreme HBL since its synchrotron peak shifts to up to two orders of magnitude higher energies during flares [2]. Thus, a flux dependent spectral hardening has been observed in both X-rays and in the GeV-TeV band (e.g. [2, 3, 4]). Because of these characteristics, Mrk 501 has been observed extensively at VHE, often during multiwavelength (MWL) campaigns to try to characterize its SED with simultaneous data, which are of primary importance for such a rapidly variable source. In 1997, it was found in a high state with fluxes $\gtrsim 1$ Crab Unit (c.u.) for several months (e.g. [5, 6, 7, 8, 9, 3]) and the historically highest VHE flux $\left(\mathrm{F}(>250 \mathrm{GeV}) \sim 8.3 \cdot 10^{-10} \mathrm{~cm}^{-2} \mathrm{~s}^{-1}\right)$ was recorded on April 16, 1997 [3]. Such a prolonged high state has not been registered since then, but important flaring activities have been observed on a few occasions, like in 2005 [10], 2012 [11] and 2014 (this work).

\section{Observations and Analysis}

H.E.S.S. The H.E.S.S. observations have been carried out in four different epoches between 2004 and 2014. The dataset comprises 34 runs $^{1}$ taken in wobble mode with the HESS I array in 2004 (4), 2006 (5) and 2012 (4) and with the full HESS II array in 2014 (21). The 2004 and 2006 runs were part of MWL campaigns and were taken on the nights of June 15-16, 2004 and July 18-19, 2006. Due to the low state of the source, only upper limits could be derived in the original analysis and the results were published separately [12, 13]. The 2012 and 2014 observations were triggered by flaring states in the $\mathrm{TeV}$ domain detected by MAGIC and FACT, respectively. In 2012 two runs in two consecutive nights were taken (June 11 and 12), while in 2014 Mrk 501 was observed on several nights between June 19-25 and on July 29-30.

In order to have an homogeneous dataset, only events detected with the CT1-4 telescopes were extracted from the 2014 observations, discarding the information coming from CT5. All but four runs pass the standard quality cuts, for a total livetime of $13.1 \mathrm{~h}(12.1 \mathrm{~h}$ when corrected for acceptance). The mean zenith angle is $63.7^{\circ}$ and the mean offset from the pointing position $0.50^{\circ}$. Data reduction has been performed using the Model analysis [14] with Loose cuts, chosen to lower the energy threshold as much as possible. The Reflected Region Background method [15] was used for the background determination of the spectral analysis. An excess of more than 2600 photons is detected with high significance. Thanks to the higher sensitivity of the Model analysis, Mrk 501 is detected also in the years 2004 and 2006. Spectral analyses have been carried out on different data subsets using the forward-folding technique [16] and three different spectral shapes.

RXTE and Swift-XRT. X-ray coverage has been ensured by RXTE and Swift-XRT. The lightcurve of the former has been retrieved from [17], while the data of the latter collected in WT

\footnotetext{
${ }^{1}$ The term "run" refers to a single observation, with a typical exposure of 28 minutes.
} 
mode between May 12 and July 30, 2012 (obsIDs: 00030793177-00030793201) and between May 31 and June 28, 2014 (obsIDs: 00035023035-00035023047) have been analysed for this work. The HEASoft software package v. $6.16^{2}$ with CALDB v. 20140120 has been used. All the events were cleaned and calibrated using the xrtpipeline task and the data in the energy range $0.3-10 \mathrm{keV}$ with grades 0-2 were analysed. The flux values for the lightcurve (Fig. 1) were calculated from the spectra of single snapshots integrating between 2 and $10 \mathrm{keV}$. These were derived in the following way: the data were grouped using the grappha tool to have a minimum of 30 counts/bin and then fit using XSPEC v. 12.8.2 with a single power-law model and Galactic hydrogen absorption fixed to $n_{H}=1.58 \cdot 10^{20} \mathrm{~cm}^{-2}$ [18]. A clear harder-when-brighter behaviour is detected, as also expected from the literature (e.g. [2]). Contemporaneous X-rays and H.E.S.S. observations exist for some nights, and are typically offset by $\sim 90$ minutes. On June 12, 2012, Swift-XRT observations start immediatly after the H.E.S.S. ones have finished.

Abastunami Observatory. Optical data are obtained with the $70 \mathrm{~cm}$ telescope of the Abastunami Observatory (Georgia), equipped with an Apogee 6E camera (between 1997-2006 a SBIG ST-6 camera). Observations taken with a R Cousins filter cover the 1997-2014 period and have been analyzed with the Daophot II reduction software using an aperture diameter of $10^{\prime \prime}$. Some nights of contemporaneous data with H.E.S.S. exist, and strictly simultanous observations have been taken during the flare peak on June 23, 2014.

\section{Results and discussion}

Lightcurves. In the top panel of Fig. 1, the run-wise lightcurve of the 2004-2014 H.E.S.S. observations above $2 \mathrm{TeV}$ is shown. Two clear flaring events can be seen in 2012 and in 2014, along with two quiescent states in 2004 and 2006 and a phase of moderate activity in 2014. Mrk 501 is clearly variable also in X-rays (middle panel) and in optical (lower panel). The comparison of the three lightcurves shows no obvious correlation in the flux variations of the different energy bands. The optical emission is in an average/low state during both $\mathrm{TeV}$ flares and this is true also for the strictly simultaneous observations during the peak of the flare in 2014. This can be explained by the harder-when-brighter behaviour detected in X-rays, since the variability at lower energy has a lower amplitude. On the other hand, the source is in high optical state in 2004 and 2006, when the TeV emission is very low. This suggests that at least two zones or mechanisms are necessary to explain the flaring events in Mrk 501. In X-rays, the brightest event is recorded in the night of June 20-21, 2014 (MJD 56829), but does not show a correspondent maximum at VHE, as might be expected. Given the rapid variability in both energy bands, this could be explained by the non-simultaneity of the two observations. The TeV flux is roughly constant during this night and is $\sim 30 \%$ higher than the one recorded on June 12, 2012. On this date, almost simultaneous X-ray observations show a $\sim 55 \%$ lower X-ray flux. Assuming an SSC emission model, a quadratic correlation between the two energy bands is expected. Thus, the source should be able to increase its $\gamma$-ray emission of almost a factor 4 in $\sim 90$ minutes. This can easily be acieved thanks to a flux doubling timescale shorter than 10 minutes [19]. A similar behaviour of high X-ray and low $\gamma$-ray emission is detected on the night of June $22-23,2014$. In this case, though, the TeV flux roughly doubles in the $\sim 2$ hours

\footnotetext{
${ }^{2}$ http://heasarc.gsfc.nasa.gov/docs/software/lheasoft
} 


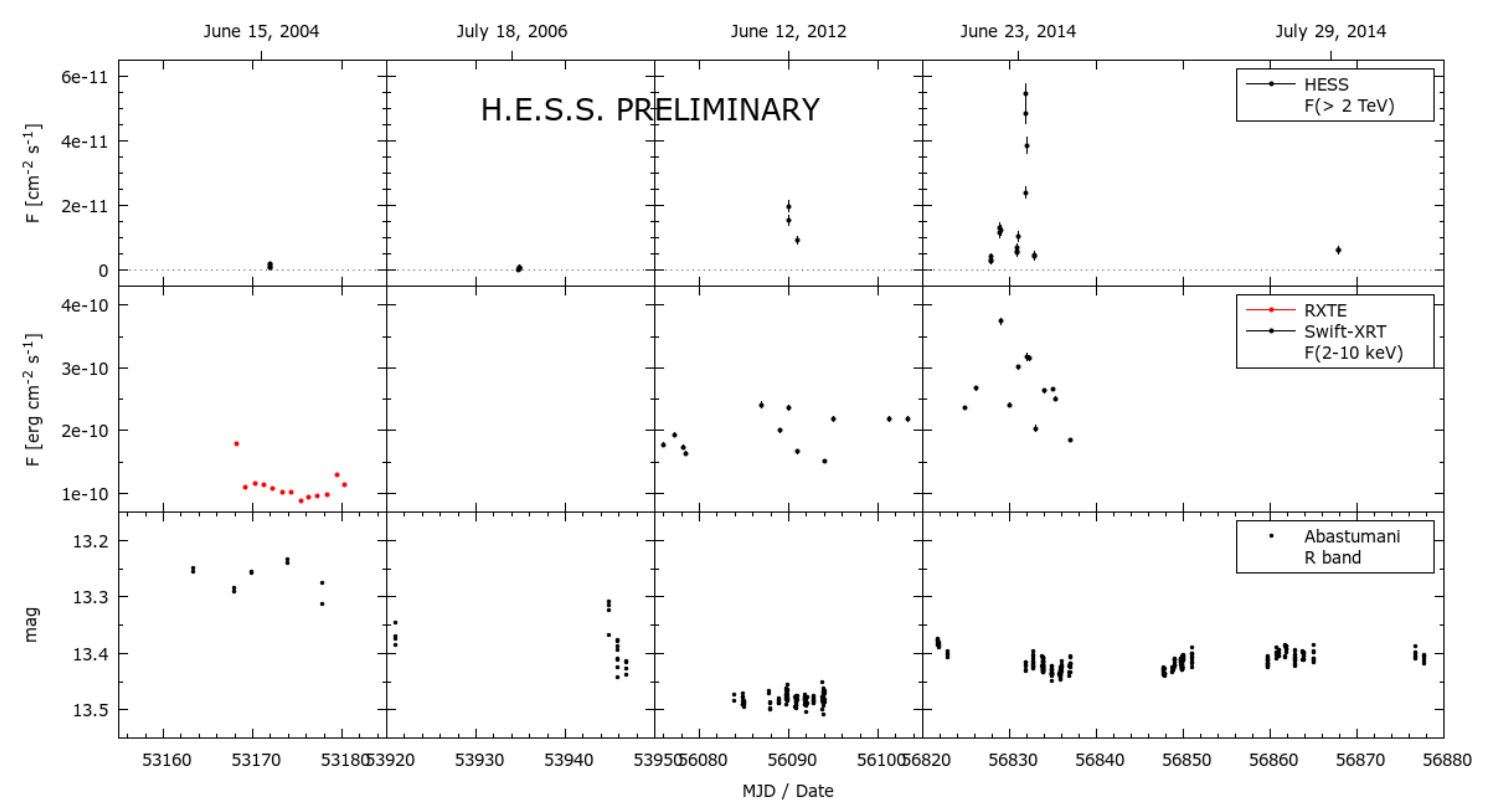

Figure 1: Top: H.E.S.S. run-wise lightcurve above $2 \mathrm{TeV}$. The flaring states in 2012 and 2014 are clearly visible (MJD 56089-90 and 56831-32). It is also apparent that the "low" state in 2014 still represent a state of moderate activity when compared to the flux levels in 2004 and 2006. Middle: RXTE and Swift-XRT X-ray lightcurves in the energy range $2-10 \mathrm{keV}$ showing high variability. Bottom: optical lightcurve from the Abastunami Observatory. No particularly high flux values or strong variability are detected in correspondence of the 2012 and $2014 \mathrm{TeV}$ flares.

of observations, suggesting a further increase to match the X-ray flux state. The opposite behaviour is registered during the TeV flare on June 23-24, 2014 (MJD 56831). The X-ray emission is 20\% lower than the maximum reached three nights before, while the $\gamma$-ray flux is $\sim 4$ times higher.

More details on the flux variability in 2014 can be found in [19].

TeV spectra. The very different flux states and high significance of the detection enable a temporally resolved spectral analysis down to very short timescales. Flux dependent spectral variability is described in the literature (e.g., $[3,10]$ ), although it has been determined mostly at energies below $2 \mathrm{TeV}$. Analyses of several datasets have been performed (total dataset, single nights and single runs during the flares) and the 2012 and 2014 spectra are shown in Fig. 2 (left). Power law (PL) spectral fits provide in general poor results and are acceptable only for low-flux nights and for some run-wise analysis. This is expected from the curved spectra described in the literature (e.g. $[3,8,10]$ ) and from the consideration that the intrinsic emitted spectrum must have been absorbed through the interaction with the extragalactic background light (EBL). Mrk 501 spectrum extends up to $\sim 20 \mathrm{TeV}$. Despite being a close source, a significant optical depth for EBL absorption $\tau \gtrsim 1$ is expected. Hence, although the curved power law (CPL) and exponential cut-off power law (ECPL) spectral shapes fit the data satisfactorily, they are not a good description of the intrinsic spectra. For this reason, in order to determine the source intrinsic spectra in the various flux states, fits including EBL absorption have been performed. The model of [20] has been used. The spectra 

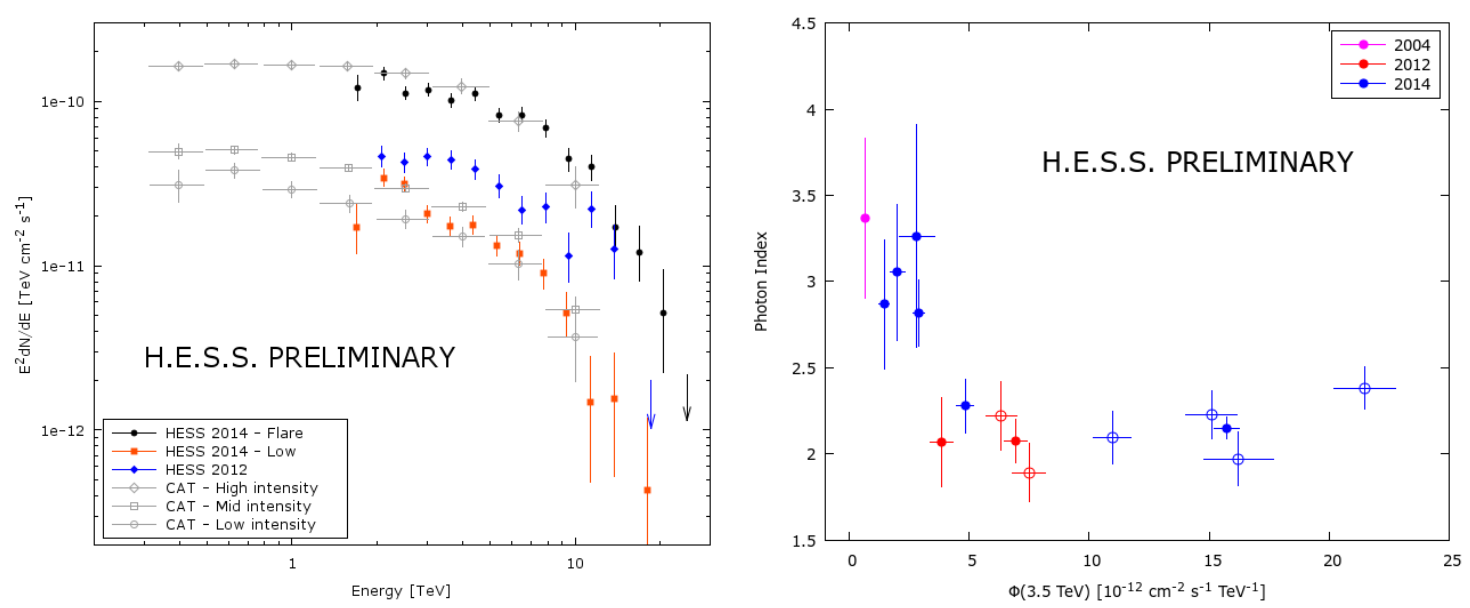

Figure 2: Left: H.E.S.S. observed spectra for the 2014 and 2012 flare and low states. The 1997 CAT spectra [3] are also shown for comparison. Right: Intrinsic photon indices from EBL absorbed PL fits as function of flux. Values are given for single nights (full circles) and for single runs (open circles) during the flares. By high fluxes, the spectra are clearly hard.

are best described by an EBL-absorbed PL. The photon indices are $2.3 \pm 0.1$ and $2.15 \pm 0.06$ for the 2012 and 2014 flaring states, and $2.7 \pm 0.1$ for the 2014 low state. The respective normalizations at $3.5 \mathrm{TeV}$ are $6.7 \pm 0.3,15.7 \pm 0.5$ and $2.8 \pm 0.1 \mathrm{~cm}^{-2} \mathrm{~s}^{-1} \mathrm{TeV}^{-1}$. Fits involving more complex spectral models are generally not significantly better anymore.

The inspection of the residuals of night and run fits shows, however, a possible trend towards a spectral softening at the highest energies, especially when lower fluxes are considered. The impossibility to detect an actual intrinsic curvature can be due to the too narrow energy range of about one decade covered by the spectra. On the other hand, the fact that this trend is mostly visible at lower fluxes points towards a harder-when-brighter behaviour also at these high energies. Indeed, the plot of the changes of the intrinsic photon indices as function of flux (Fig. 2, right) shows that the spectra are clearly hard during the flares. This can be explained as being in the vicinity of the maximum of the IC peak, which is known to migrate towards higher energies during flares and periods of high activity (e.g. [3, 10]). This is the first time that such behaviour is reported for energies extending significantly beyond $10 \mathrm{TeV}$, up to $20 \mathrm{TeV}$ and for such short time intervals. The hard PL intrinsic spectrum during the 2014 flare also indicates that there is no sign of being in the Klein-Nishina regime even at these high energies.

EBL determination. It is possible that the optical depth $\tau$ derived from [20] for the 2-20 $\mathrm{TeV}$ energy range at the redshift of Mrk $501(z \simeq 0.034)$ is not precise. In order to test the model, the procedure described in [21] was applied. The sum of the LogLikelihood ratio test ${ }^{3}$ (LLRT) profiles peaks at the normalization factor $\alpha=0.93_{-0.14}^{+0.15}$, with a value of $\sim 83$, which translate in a significance of $9.1 \sigma$. The null hypothesis of a non-existent EBL is hence excluded at more than $9 \sigma$ level. The derived $\alpha$ is compatible with the model of [20]. Taking into account the contributions of the two flares only, $\alpha=0.89_{-0.14}^{+0.16}$, which is not compatible with the one calculated in [21] $(\alpha=$

${ }^{3}$ LLRT $=-2 \cdot \log \left[\mathcal{L}\left(H_{0}\right) / \mathcal{L}\left(H_{1}\right)\right]$, where $H_{0}$ is the null hypothesis, i.e. $\alpha=0$, and $H_{1}$ the hypothesis to be tested, $\alpha>0$. The significance is then calculated as $\sqrt{L L R T}$. 
$1.27_{-0.15}^{+0.18}$. This can be explained by the fact that, altough several sources have been used in [21], the major contribution is given by PKS 2155-304 alone. Differences could therefore be explained as dependencies on the redshift $\left(z_{M r k 501} \simeq 0.034\right.$ vs. $\left.z_{P K S} 2155-304=0.116\right)$ and on the significantly different energy range covered ( $\sim 2-20 \mathrm{TeV}$ vs. $\sim 0.15-7 \mathrm{TeV})$. A further comparison with the lowredshift subset composed of Mrk 421 and PKS 2005-489 only $(<z>=0.051)$ is not very meaningful because of the large errors on the normalization $\left(\alpha_{l o w-z}=1.6_{-1.1}^{+0.5}\right.$, [21] $)$ and because the various subsets yield results with LLRT values between -2 and 2, hence not significant. On the other hand, the present result is perfectly compatible with the one derived in [21] for the high energy dataset $\alpha_{\text {high-energy }}=1.05_{-0.28}^{+0.32}$, which covers the range $0.95-14 \mathrm{TeV}$.

LIV studies and QG scale limits. The 2014 flare data were exploited to search for Lorentz Invariance Violation (LIV) effects leading to limits on the Quantum Gravity (QG) scale. In spite of its low redshift $(z \sim 0.034)$, Mrk 501 is a very promising source for LIV studies due to the hard energy spectrum. The study of energy dependent time-delays within a deterministic scenario and with a Likelihood method was performed following [22]. It consists of the construction of a low-energy template light-curve $(2-4.5 \mathrm{TeV})$ injected in the likelihood fit procedure with photons of energy above $4.5 \mathrm{TeV}(\sim 500$ photons with a negligible background contribution below $0.5 \%)$ and studied with dedicated simulations. Two cases were considered: a linear and a quadratic dependence of the speed of light on the photon energy. As no significant time delay was found (more than $1 \sigma$ from zero), limits on the QG scale were computed. The one-sided 95\% CL QG limits including systematic uncertainties for the sub-luminal (supra-luminal) case are $8.5 \times 10^{17} \mathrm{GeV}\left(6.4 \times 10^{17} \mathrm{GeV}\right)$ for the linear term and $1.15 \times 10^{11} \mathrm{GeV}\left(1.0 \times 10^{11} \mathrm{GeV}\right)$ for the quadratic term. The main uncertainties come from the template light-curve binned fit, energy calibration, knowledge of the acceptance corrections and of the smearing factors in energy. The total contribution of the systematic effects to the error calculation was conservatively estimated to be below $100 \%$ of the statistical one. Another type of systematics may come from the energy-dependent source effects which may lead to energy-dependent time lags, not considered in this study. As first results, these are one of best constraints on the linear term derived from AGN observations (comparable with those obtained with the PKS 2155-304 2006 flare [22]) and the best values on the quadratic term obtained until now with GRBs [23] and AGNs [22] data. This is mainly due to the exceptional strength of the Mrk 501 2014 flare leading to a high number of photons in the energy range of $10-20 \mathrm{TeV}$.

\section{Conclusion}

H.E.S.S. observations of Mrk 501 between 2004 and 2014 have been presented in a MWL context. Rapid variability in the $2-20 \mathrm{TeV}$ energy range during flaring states in 2012 and 2014 has been reported. No direct relation with the emission at lower energies has been found. While the different behaviour at X-ray energies can be in principle explained by the time offset between the observations (this would imply the capability of the source to increase its $\gamma$-ray flux of a factor $\sim$ four in $\sim 90$ minutes), the optical emission clearly does not correlate with the VHE flux. It is in low state in the strictly simultaneous observations during the 2014 flare, while it is significantly higher during the TeV low states in 2004 and 2006. This suggests the presence of at least two zones or mechanisms able to produce flaring events in this source. The TeV spectra are hard during the periods of high flux, and softer otherwise. In particular, the hard simple PL shape extending above 
$20 \mathrm{TeV}$ during the 2014 flare peak indicates the absence of Klein-Nishina suppression at these high energies. Studies on the EBL intensity exclude the hypothesis of a non-existent EBL at the $9 \sigma$ level. The normalization factors derived with all datasets $\left(\alpha=0.93_{-0.14}^{+0.15}\right)$ and with the flare datasets only $\left(\alpha=0.89_{-0.14}^{+0.16}\right)$ are consistent with the model of [20]. The quadratic limits on the QM scale $\left(1.15 \times 10^{11} \mathrm{GeV}\right)$ are the best ones derived from GRB and AGN observations.

\section{Acknowledgments}

The support of the Namibian authorities and of the University of Namibia in facilitating the construction and operation of H.E.S.S. is gratefully acknowledged, as is the support by the German Ministry for Education and Research (BMBF), the Max Planck Society, the German Research Foundation (DFG), the French Ministry for Research, the CNRS-IN2P3 and the Astroparticle Interdisciplinary Programme of the CNRS, the U.K. Science and Technology Facilities Council (STFC), the IPNP of the Charles University, the Czech Science Foundation, the Polish Ministry of Science and Higher Education, the South African Department of Science and Technology and National Research Foundation, and by the University of Namibia. We appreciate the excellent work of the technical support staff in Berlin, Durham, Hamburg, Heidelberg, Palaiseau, Paris, Saclay, and in Namibia in the construction and operation of the equipment. N.C. acknowledges support from Alexander von Humboldt foundation. This research has made use of the lightcurves provided by the University of California, San Diego Center for Astrophysics and Space Sciences, X-ray Group (R.E. Rothschild, A.G. Markowitz, E.S. Rivers, and B.A. McKim), obtained at http: //cass.ucsd.edu/ rxteagn/.

\section{References}

[1] J. Quinn et al., Detection of Gamma Rays with E > 300 GeV from Markarian 501, ApJ 456 (1996) L83.

[2] E. Pian et al., BeppoSAX Observations of Unprecedented Synchrotron Activity in the BL Lacertae Object Markarian 501, ApJ 492 (1998) L17-L20, [astro-ph/9710331].

[3] A. Djannati-Atai et al., Very High Energy Gamma-ray spectral properties of MKN 501 from CAT Čerenkov telescope observations in 1997, AEA 350 (1999) 17-24, [astro-ph/9906060].

[4] A. A. Abdo et al., Insights into the High-energy y-ray Emission of Markarian 501 from Extensive Multifrequency Observations in the Fermi Era, ApJ 727 (2011) 129, [arXiv: 1011 . 5260].

[5] M. Catanese et al., Multiwavelength Observations of a Flare from Markarian 501, ApJ 487 (1997) L143-L146, [astro-ph/9707179].

[6] F. W. Samuelson et al., The TeV Spectrum of Markarian 501, ApJ 501 (1998) L17-L20.

[7] F. Aharonian et al., Measurement of the flux, spectrum, and variability of TeV $\gamma$-rays from MKN 501 during a state of high activity., AEFA 327 (1997) L5-L8.

[8] F. A. Aharonian et al., The time averaged TeV energy spectrum of MKN 501 of the extraordinary 1997 outburst as measured with the stereoscopic Cherenkov telescope system of HEGRA, AEA 349 (1999) 11-28, [astro-ph/9903386]. 
[9] F. A. Aharonian et al., The temporal characteristics of the TeV gamma-radiation from MKN 501 in 1997. I. Data from the stereoscopic imaging atmospheric Cherenkov telescope system of HEGRA, AEA 342 (1999) 69-86, [astro-ph/9808296].

[10] J. Albert et al., Variable Very High Energy $\gamma$-Ray Emission from Markarian 501, ApJ 669 (2007) 862-883, [astro-ph/0702008].

[11] F. Borracci et al., Magic results and multi-wavelength observations of a mrk 501 flare in june 2012, in Proceedings of the 33rd ICRC, Rio de Janeiro, Brazil, 2013.

[12] F. Aharonian et al., Observations of selected AGN with HESS, AEA 441 (2005) 465-472, [astro-ph/0507207].

[13] F. Aharonian et al., Upper limits from HESS active galactic nuclei observations in 2005-2007, AEA 478 (2008) 387-393, [arXiv : 0711.3196].

[14] M. de Naurois and L. Rolland, A high performance likelihood reconstruction of $\gamma$-rays for imaging atmospheric Cherenkov telescopes, Astroparticle Physics 32 (2009) 231-252, [arXiv: 0907 . 2610].

[15] D. Berge, S. Funk, and J. Hinton, Background modelling in very-high-energy $\gamma$-ray astronomy, AEA 466 (2007) 1219-1229, [astro-ph/0610959].

[16] F. Piron et al., Temporal and spectral gamma-ray properties of Mkn 421 above $250 \mathrm{GeV}$ from CAT observations between 1996 and 2000, AEFA 374 (2001) 895-906, [astro-ph/0106196].

[17] E. Rivers, A. Markowitz, and R. Rothschild, Full Spectral Survey of Active Galactic Nuclei in the Rossi X-ray Timing Explorer Archive, ApJ 772 (2013) 114, [arXiv: 1306. 4376].

[18] P. M. W. Kalberla et al., The Leiden/Argentine/Bonn (LAB) Survey of Galactic HI. Final data release of the combined LDS and IAR surveys with improved stray-radiation corrections, AEA 440 (2005) 775-782, [astro-ph/0504140].

[19] N. Chakraborty et al., Rapid variability at very high energies in Mrk 501, in proceedings of 34th International Cosmic Ray Conference (ICRC2015), PoS (ICRC2015) 872, 2015.

[20] A. Franceschini, G. Rodighiero, and M. Vaccari, Extragalactic optical-infrared background radiation, its time evolution and the cosmic photon-photon opacity, AEGA 487 (2008) 837-852, [arXiv:0805. 1841].

[21] H.E.S.S. Collaboration et al., Measurement of the extragalactic background light imprint on the spectra of the brightest blazars observed with H.E.S.S., AEA 550 (2013) A4, [arXiv: 1212 . 3409].

[22] H.E.S.S. Collaboration et al., Search for Lorentz Invariance breaking with a likelihood fit of the PKS 2155-304 flare data taken on MJD 53944, Astroparticle Physics 34 (2011) 738-747, [arXiv: 1101.3650].

[23] V. Vasileiou et al., Constraints on Lorentz invariance violation from Fermi-Large Area Telescope observations of gamma-ray bursts, Phys. Rev. D 87 (2013), no. 12 122001, [arXiv: 1305. 3463]. 